OPEN ACCESS

Edited by:

Xuejun Wang,

University of South Dakota,

United States

Reviewed by:

DeWayne Townsend,

University of Minnesota Twin Cities,

United States

Atsushi Kuno,

Sapporo Medical University, Japan Jun Tanihata,

Jikei University School of Medicine,

Japan

*Correspondence: Joshua T. Selsby

jselsby@iastate.edu

Specialty section:

This article was submitted to

Striated Muscle Physiology,

a section of the journal

Frontiers in Physiology

Received: 05 April 2021 Accepted: 03 June 2021

Published: 09 July 2021

Citation:

Krishna S, Spaulding HR,

Quindry TS, Hudson MB, Quindry JC

and Selsby JT (2021) Indices

of Defective Autophagy in Whole

Muscle and Lysosome Enriched

Fractions From Aged D2-mdx Mice.

Front. Physiol. 12:691245.

doi: 10.3389/fphys.2021.691245

\section{Indices of Defective Autophagy in Whole Muscle and Lysosome Enriched Fractions From Aged D2-mdx Mice}

\author{
Swathy Krishna', Hannah R. Spaulding', Tiffany S. Quindry2, Matthew B. Hudson², \\ John C. Quindry ${ }^{2}$ and Joshua T. Selsby ${ }^{1 *}$ \\ ${ }^{1}$ Department of Animal Science, lowa State University, Ames, IA, United States, ${ }^{2}$ School of Integrative Physiology \\ and Athletic Training, University of Montana, Missoula, MT, United States, ${ }^{3}$ Department of Kinesiology and Applied \\ Physiology, University of Delaware, Newark, DE, United States
}

Duchenne muscular dystrophy (DMD) is a fatal, progressive muscle disease caused by the absence of functional dystrophin protein. Previous studies in mdx mice, a common DMD model, identified impaired autophagy with lysosomal insufficiency and impaired autophagosomal degradation as consequences of dystrophin deficiency. Thus, we hypothesized that lysosomal abundance would be decreased and degradation of autophagosomes would be impaired in muscles of D2-mdx mice. To test this hypothesis, diaphragm and gastrocnemius muscles from 11 month-old D2-mdx and DBA/2J (healthy) mice were collected. Whole muscle protein from diaphragm and gastrocnemius muscles, and protein from a cytosolic fraction (CF) and a lysosomeenriched fraction (LEF) from gastrocnemius muscles, were isolated and used for western blotting. Initiation of autophagy was not robustly activated in whole muscle protein from diaphragm and gastrocnemius, however, autophagosome formation markers were elevated in dystrophic muscles. Autophagosome degradation was impaired in D2-mdx diaphragms but appeared to be maintained in gastrocnemius muscles. To better understand this muscle-specific distinction, we investigated autophagic signaling in CFs and LEFs from gastrocnemius muscles. Within the LEF we discovered that the degradation of autophagosomes was similar between groups. Further, our data suggest an expanded, though impaired, lysosomal pool in dystrophic muscle. Notably, these data indicate a degree of muscle specificity as well as model specificity with regard to autophagic dysfunction in dystrophic muscles. Stimulation of autophagy in dystrophic muscles may hold promise for DMD patients as a potential therapeutic, however, it will be critical to choose the appropriate model and muscles that most closely recapitulate findings from human patients to further develop these therapeutics.

Keywords: dystrophin, Duchenne muscular dystrophy, mouse model, diaphragm, gastrocnemius, D2-mdx 


\section{INTRODUCTION}

Duchenne muscular dystrophy (DMD) is a progressive muscledegenerative disease caused by the absence of functional dystrophin protein (Hoffman et al., 1987). Dystrophin plays an important role in maintaining structural stability by establishing a link between cytoskeletal actin and the extracellular matrix (Petrof et al., 1993b; Ehmsen et al., 2002). Dystrophin deficiency causes disruption of the dystrophin associated protein complex (DAPC) and leads to sarcolemmal fragility particularly during eccentric contractions (Petrof et al., 1993b). The resultant cellular injuries and altered signaling originating from the DAPC (Rahimov and Kunkel, 2013; Houang et al., 2018) initiate a cascade of malfunction in muscle fibers (Selsby et al., 2010, 2012; Selsby, 2011; Spaulding et al., 2018) that ultimately cause injury, inflammation, fibrosis, and fatty infiltration at the whole muscle level (Chen et al., 2005; Morris et al., 2010).

In cells from healthy muscle, alterations to autophagic flux can maintain homeostasis during cell stress or injury (Sandri, 2010; Castets et al., 2016). During autophagy, damaged organelles and proteins are sequestered in autophagosomes and then subjected to lysosomal degradation (reviewed in Parzych and Klionsky, 2014; Farre and Subramani, 2016; Kocaturk and Gozuacik, 2018). We and others have reported defective autophagy and increased autophagosome efflux, termed autophagosome escape, in dystrophin-deficient muscle from $\mathrm{mdx}$ mice and the limited data from human DMD patients suggest similar outcomes (De Palma et al., 2012; Pauly et al., 2012; Spitali et al., 2013; Castets et al., 2016; Spaulding et al., 2018, 2020a). These data raise the possibility that stimulation of autophagy or degradation of autophagosomes may be effective therapeutic strategies for DMD (Hollinger and Selsby, 2013; Pal et al., 2015; Spaulding et al., 2020a). Indeed, pharmacological activation of AMP-activated protein kinase (AMPK), a known activator of autophagy, and stimulation of transcription factor EB (TFEB), a transcription factor that drives transcription of autophagic machinery, implicates dysfunctional autophagy as part of the dystrophic disease process, but also demonstrates the potential therapeutic value of increased autophagy for dystrophin deficiency (Ljubicic et al., 2012; Pauly et al., 2012; Spaulding et al., 2020a).

Autophagic degradation is facilitated by lysosomal enzymes including a variety of proteases and hydrolases (Saftig and Klumperman, 2009; Ballabio, 2016). The efficiency and effectiveness of lysosomal degradation of autophagosomes can be influenced by lysosome abundance, size, $\mathrm{pH}$, hydrolase content, localization, and fusion (Settembre and Ballabio, 2011; Roczniak-Ferguson et al., 2012). We found previously that lysosome abundance was decreased in dystrophic muscle from mdx mice (Spaulding et al., 2018). Additionally, a large percentage of those lysosomes were observed in the extracellular space, likely affiliated with infiltrating immune cells, which would further diminish the predicted degradative power of the lysosomal pool in dystrophic muscle fibers (Spaulding et al., 2018). We also discovered that increased lysosomal abundance was associated with decreased disease severity in dystrophic muscle (Spaulding et al., 2020a). In addition, up-regulation of Src kinase may also impair autophagosome and lysosome fusion in dystrophic muscle, which would limit degradation of autophagosomal cargo (Pal et al., 2015).

Coinciding with increased interest in autophagic dysfunction in dystrophic muscle was the emergence of the D2-mdx model. The relatively novel D2-mdx mouse model was produced by backcrossing mdx mice onto the DBA background and appears to produce a more severe dystrophinopathy model compared to the conventional $m d x$ mouse (Coley et al., 2016; van Putten et al., 2019). Mutations in the latent TGF $\beta$ binding protein gene (LTBP) cause increased fibrosis and in the Anxa6 gene result in decreased muscle repair in the DBA background, in addition to the nonsense mutation in exon 23 of dystrophin gene, were attributed for the severity of this model compared to the $\mathrm{mdx}$ model (Coley et al., 2016; van Putten et al., 2019). Given the potential importance of this new model for therapeutic development (Hammers et al., 2020), the purpose of this study was to perform an initial exploration of autophagosome degradation in the D2-mdx model. We hypothesized that, similar to $\mathrm{mdx}$ mice, dystrophin deficiency in D2-mdx mice would cause decreased lysosomal abundance and accumulation of autophagosomes.

\section{MATERIALS AND METHODS}

\section{Animal Treatments}

All animal procedures were approved by the Institutional Animal Care and Use Committees at the University of Montana and the University of Florida. Animals were fed ad libitum. Functional and histological data from the diaphragm and limb muscles from these mice has been previously published (Spaulding et al., 2019b, 2020b). Briefly, 11 month old male D2.B10-Dmdmdx/J (D2-mdx; dystrophin-deficient) and male DBA-2J (DBA; healthy control) mice were sedated to a surgical level of anesthesia, the gastrocnemius muscles and diaphragms removed, and mice were euthanized via exsanguination. Muscles were snap frozen in liquid nitrogen for subsequent analyses.

\section{Protein Isolation}

To extract the whole homogenate, diaphragm and gastrocnemius muscles were powdered on dry ice and protein was extracted from 30 to $65 \mathrm{mg}$ of muscle using a 1:10 weight to volume ratio in whole muscle extraction buffer $(10 \mathrm{mM}$ sodium phosphate buffer, $\mathrm{pH} 7.0,2 \% \mathrm{SDS}, 1 \%$ Halt $^{\mathrm{TM}}$ protease and phosphatase inhibitor single-use cocktail) using a hand-held homogenizer. Samples were centrifuged for $15 \mathrm{~min}$ at $1,500 \times g$ and the supernatant collected in a microcentrifuge tube.

The cytosolic (CF) and lysosome-enriched fractions (LEF) were obtained from protein extracted from gastrocnemius muscles using a lysosomal isolation kit from Sigma-Aldrich (St. Louis, MO, LYSISO1) according to manufacturer instructions with minor modifications. Briefly, approximately $25 \mathrm{mg}$ of powdered, frozen muscle was homogenized in a 1:4 weight to volume ratio in lysosomal extraction buffer (LEB) containing $1 \%$ protease inhibitor and centrifuged at $1,000 \times g$ for $10 \mathrm{~min}$. The resultant supernatant was collected and saved on ice. The pellet was resuspended in 2 volumes $\mathrm{LEB} / \mathrm{mg}$ initial weight of sample, centrifuged again as above, and the supernatant was collected and 
pooled with the first supernatant. This process was repeated once more and the supernatant added to the pooled supernatants from the first two steps. The pooled supernatant was then centrifuged at $20,000 \times g$ for $20 \mathrm{~min}$. The supernatant from this step was considered the $\mathrm{CF}$ and was transferred to a fresh tube and stored at $-80^{\circ} \mathrm{C}$. The pelleted portion was considered the LEF, which was resuspended in LEB at $0.8 \mathrm{~mL} / \mathrm{g}$ of original tissue weight and stored at $-80^{\circ} \mathrm{C}$ until use. We confirmed LEF by measuring relative protein abundance of the lysosomal membrane protein, LAMP2, in both the LEF and CF using western blot (described below). LAMP2 was only detectable in the LEF.

\section{Western Blots}

Protein concentration from whole muscle homogenate and extracted fractions were quantified using a Pierce BCA Protein Assay kit (Thermo Scientific) and diluted with Laemmli buffer (Bio-Rad), denatured at $95^{\circ} \mathrm{C}(5 \mathrm{~min})$, and separated on a $4-20 \%$ precast gel (Bio-Rad; $60 \mathrm{~V}$ for $30 \mathrm{~min}$ and $120 \mathrm{~V}$ for $70 \mathrm{~min})$. Protein from whole homogenates was diluted to $4 \mu \mathrm{g} / \mu \mathrm{L}$ and $40 \mu \mathrm{g}$ protein was loaded into each lane. To enable comparisons between fractions, fractions were brought to an equal concentration of $0.8 \mu \mathrm{g} / \mu \mathrm{L}$ and $8 \mu \mathrm{g}$ protein was loaded into each well on each gel. Protein was transferred to a nitrocellulose membrane at $100 \mathrm{~V}$ for $1 \mathrm{~h}$. Following transfer, equal protein loading was confirmed by Ponceau$\mathrm{S}$ staining. Ponceau-S-stained membranes were imaged and objectively quantified using AzureSpot software version 2.0.062. Total optical density did not differ between groups for any membrane though the banding pattern differed between the LEF and the CF. The membranes were rinsed in Tris buffered saline with $0.1 \%$ Tween 20 (TBST, 2 times, 2 min each) to remove Ponceau-S stain and then blocked in 5\% milk (5 g nonfat dry milk in $100 \mathrm{~mL}$ of TBST) for $1 \mathrm{~h}$. After blocking, the membranes were rinsed in TBST (2 times, 2 min each) and incubated in primary antibodies at $4^{\circ} \mathrm{C}$ overnight (described below), washed in TBST (3 times, $10 \mathrm{~min}$ each), incubated in secondary antibodies at room temperature for $1 \mathrm{~h}$, washed in TBST (3 times, $10 \mathrm{~min}$ ) and then incubated in ECL (BioRad Clarity ${ }^{\mathrm{TM}}$ ) for $6 \mathrm{~min}$ in the dark. Membranes were imaged using an Azure ${ }^{\mathrm{TM}} \mathrm{C} 600$ and bands were objectively measured using AzureSpot software. The lanes were identified and marked and then the bands were detected and quantified using automated band detection where possible to limit bias that may occur.

The following dilutions of antibodies from Cell Signaling (Danvers, MA) (unless otherwise noted) were used to probe membranes containing diaphragm and gastrocnemius whole muscle proteins: AMPK [Primary (P) 1:1000 TBST, Secondary (S) 1:2000 5\% milk, Product number 5832S], pAMPK T172 (P 1:500 TBST, S 1:2000 TBST, 2535S), ULK1 (P 1:500 TBST, S 1:2000 TBST, 8054S), pULK1 S555 (P 1:500 TBST, S 1:2000 5\% milk, 5869S), Beclin1 (P 1:500 5\% milk, S 1:2000 5\% milk, 3495S), pBeclin1 S91 (P 1:500 5\% milk, S 1:2000 5\% milk, 14717S), PI3K class III (P 1:1000 1\% milk, S 1:1000 5\% milk, 3358S), LAMP2 (P 1:1000 TBST, S 1:2000 5\% milk, 49067S), Cathepsin B (P 1:1000 TBST, S 1:2000 5\% milk, 31718S), p62 (Abcam, P 1:500 5\% milk, S 1:1000 5\% milk, ab56416), ATG12 (P 1:1000 TBST, S 1:2000 TBST,
4180S also used to detect ATG12-ATG5), and LC3A/B (P 1:500 5\% milk, S 1:1000 5\% milk, 12741S).

Several additional proteins were probed in whole muscle homogenates from diaphragm: ATG7 (P 1:1000 1\% milk, S 1:1000 5\% milk, 8558S), ATG16L1 (P 1:1000 1\% milk, S 1:1000 5\% milk, 8089S) and LAMP1 (P 1:1000 TBST, S 1:2000 5\% milk, 3243S).

CF proteins from gastrocnemius muscles were probed using: AMPK [Primary (P) 1:1000 TBST, Secondary (S) 1:2000 5\% milk, 5832S], pAMPK T172 (P 1:500 TBST, S 1:2000 TBST, 2535S), ULK1 (P 1:500 TBST, S 1:2000 TBST, 8054S), pULK1 S555 (P 1:500 TBST, S 1:2000 5\% milk, 5869S), Beclin1 (P 1:500 5\% milk, S 1:2000 5\% milk, 3495S), pBeclin1 S91 (P 1:500 5\% milk, S 1:2000 5\% milk, 14717S), PI3K class III (P 1:1000 1\% milk, S 1:1000 5\% milk, 3358S), LAMP2 (P 1:1000 TBST, S 1:2000 5\% milk, 49067S), Cathepsin B (P 1:1000 TBST, S 1:2000 5\% milk, 31718S), p62 (Abcam, P 1:500 5\% milk, S 1:1000 5\% milk, ab56416), ATG12 (P 1:1000 TBST, S 1:2000 TBST, 4180S, also used to detect ATG12-ATG5), and LC3A/B (P 1:500 5\% milk, S 1:1000 5\% milk, 12741S).

To probe LEF we used: LAMP2 (P 1:1000 TBST, S 1:2000 TBST, 49067S), Cathepsin B (P 1:1000 TBST, S 1:2000 TBST, 31718S), p62 (Abcam, P 1:500 2.5\% milk, S 1:1000 5\% milk, ab56416), ATG12 (P 1:1000 2.5\% milk, S 1:2000 2.5\% milk, 4180S), LC3A/B (P 1:500 2.5\% milk, S 1:1000 5\% milk, 12741S) and ATG16L1 (P 1:1000 TBST, S 1:2000 TBST, 8089S).

\section{Statistics}

Data were analyzed using a $2 \times 2$ ANOVA with main effects of disease and fraction as well as the disease $\mathrm{x}$ fraction in instances where probed proteins were detected in LEF and CF of both healthy and dystrophic muscle followed by a Tukey post hoc test. When bands were only detected in a single fraction and for whole muscle homogenate from diaphragm or gastrocnemius muscles, a Student's unpaired T-test was used. All statistical analyses were performed using GraphPad Prism [version 8.2.0(272)] and significance was established at $p<0.05$.

\section{RESULTS}

In previous work we established histological injury in diaphragms used in this study. Specifically, we discovered that in 11 months old D2-mdx mice fibrosis was increased by approximately 5 -fold compared to healthy controls. Further, we also discovered specific tension was decreased by approximately 50\% compared to healthy controls (Spaulding et al., 2020b). Similarly, in limb muscles from these D2-mdx, tetanic force and specific tension in soleus were both decreased by $30 \%$ and in EDL were decreased by 50 and $20 \%$, respectively, compared to DBA. Fibrotic area in limb muscles increased from less than $5 \%$ in DBA to $11 \%$ in D2-mdx (Spaulding et al., 2019b).

\section{Markers of Autophagic Processes Differ Between Dystrophin-Deficient Diaphragms and Gastrocnemius Muscles}

To understand how markers of autophagy were impacted by dystrophin deficiency in the D2-mdx model, we measured 
relative protein abundance of activation, formation, and degradation markers in diaphragms and gastrocnemius muscles from 11 month old, male animals with advanced disease. In the diaphragm, total AMPK increased 3.5-fold $(p<0.0001)$ in D2-mdx compared to DBA, pAMPK (phosphorylated at T172) was similar between groups, and the pAMPK T172/AMPK was decreased by $80 \%$ with disease $(p<0.05$; Figure 1). Likewise, total ULK1 was increased 70\% $(p<0.01)$ in diseased diaphragms, however, pULK1 (phosphorylated at S555) and pULK1 S555/ULK1 were similar between groups (Figure 1). Total Beclin1 was increased 10.7-fold $(p<0.0001)$ and pBeclin 1 (phosphorylated at S91) was increased 3.8-fold $(p<0.0001)$ in D2-mdx diaphragms compared to diaphragms from DBA, resulting in a $70 \%(p<0.05)$ reduction of pBeclin1 S91/total Beclin1 in dystrophic diaphragms (Figure 1). PI3 kinase class III (PI3K III) was increased by approximately 3-fold $(p<0.0001)$ in diaphragms from D2-mdx compared to diaphragms from DBA (Figure 1).

The relative abundance of autophagosome formation markers ATG7 $(p<0.001)$ and ATG12-5 $(p<0.001)$ was increased approximately 2 -fold and ATG16L1 was increased by 3.7 -fold $(p<0.001)$ in muscles from D2-mdx compared to DBA (Figure 1). LC3A/B I was increased by 3.6-fold $(p<0.0001)$ and the relative abundance of LC3A/B II, an autophagosome marker, was increased 2 -fold $(p<0.05)$ in dystrophic muscle compared to healthy, while the LC3A/B II/I was similar between groups (Figure 1). The relative abundance of autophagic degradation marker p62 increased 2.7-fold $(p<0.01)$ in D2-mdx diaphragms compared to diaphragms from DBA mice (Figure 1). The lysosomal membrane proteins LAMP1 and LAMP2 were similar between groups (Figure 1).

To better understand the uniformity of autophagic dysfunction within different muscles from the same animals, we also probed autophagy in gastrocnemius muscles. Total AMPK was increased by 3.8 -fold $(p<0.05)$, and pAMPK T172 6 -fold $(p<0.0001)$, in dystrophic muscle; however, the ratio of pAMPK T172/AMPK was similar between groups (Figure 2). Total ULK1 was similar between healthy and dystrophic muscles, however, pULK1 S555 was decreased by 0.5 -fold $(p<0.05)$ in D2-mdx compared to DBA and the ratio was similar between the two groups (Figure 2). Total Beclin1 was increased by $72 \%$ $(p<0.01)$ and pBeclin1 S91 was increased by $89 \%(p<0.01)$

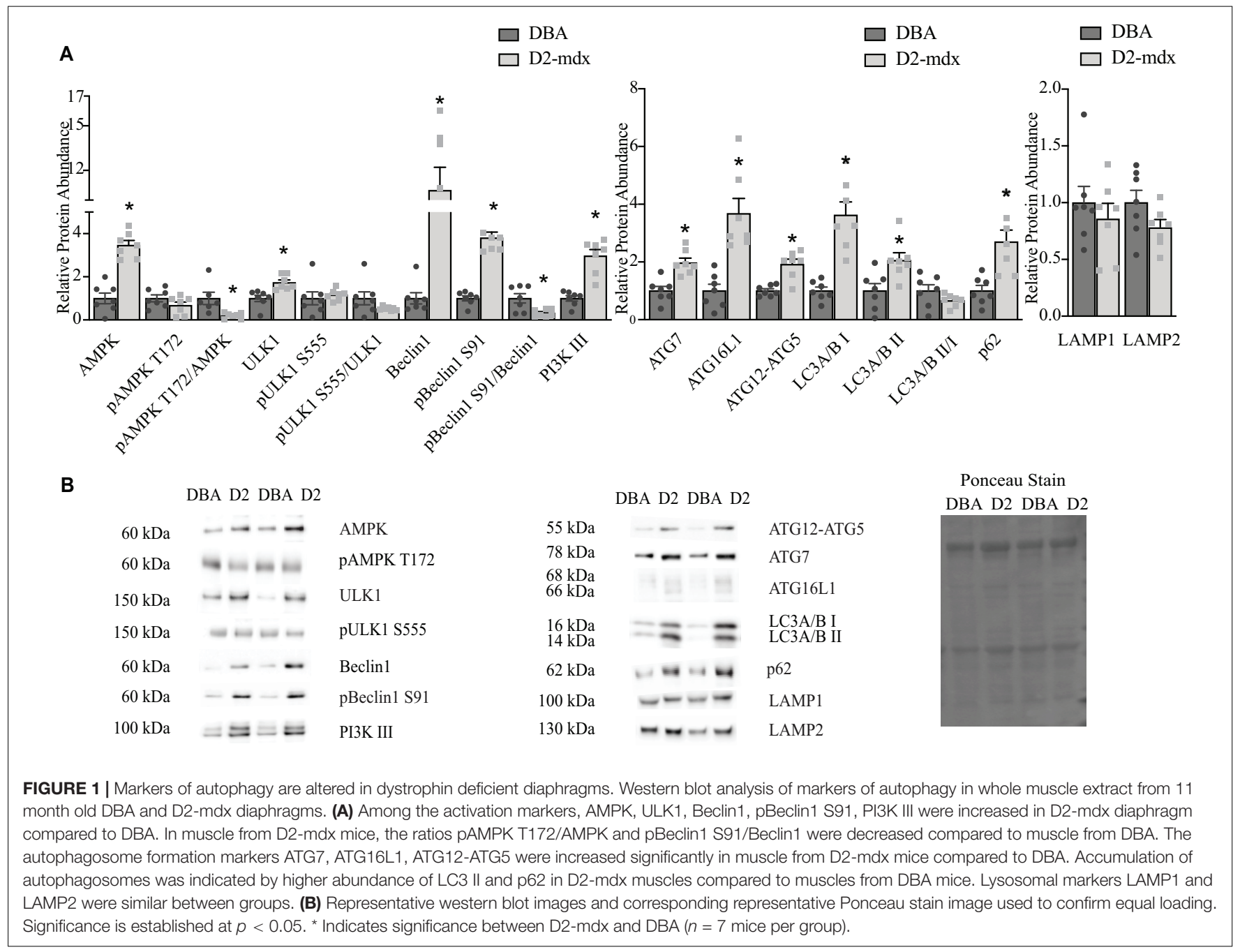




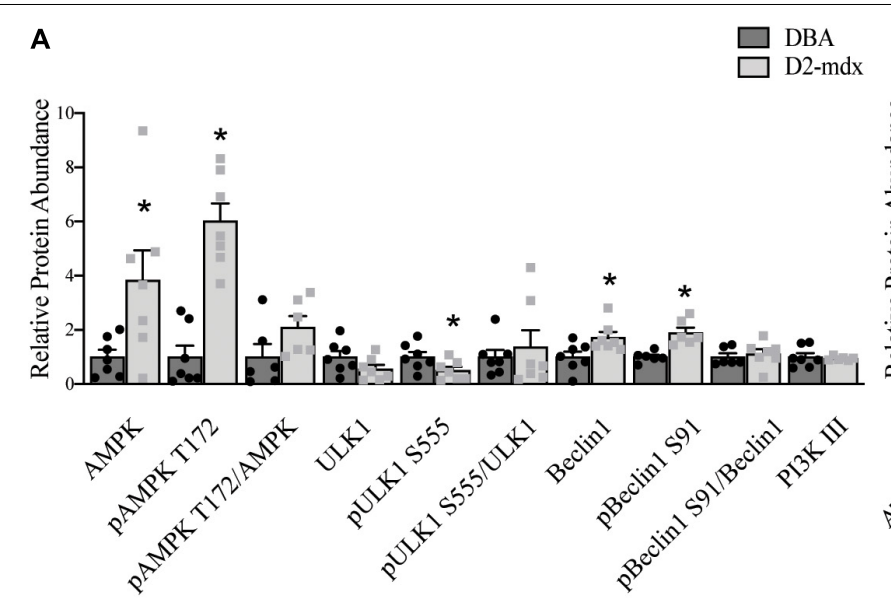

B
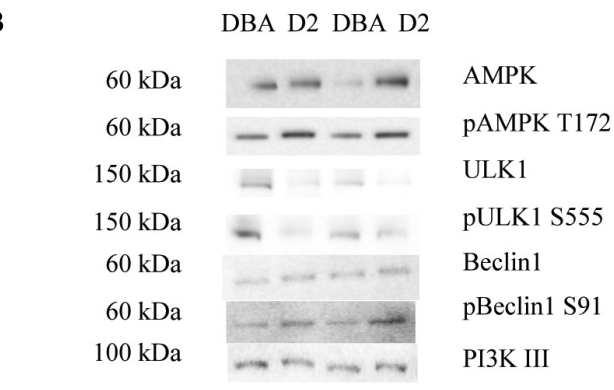

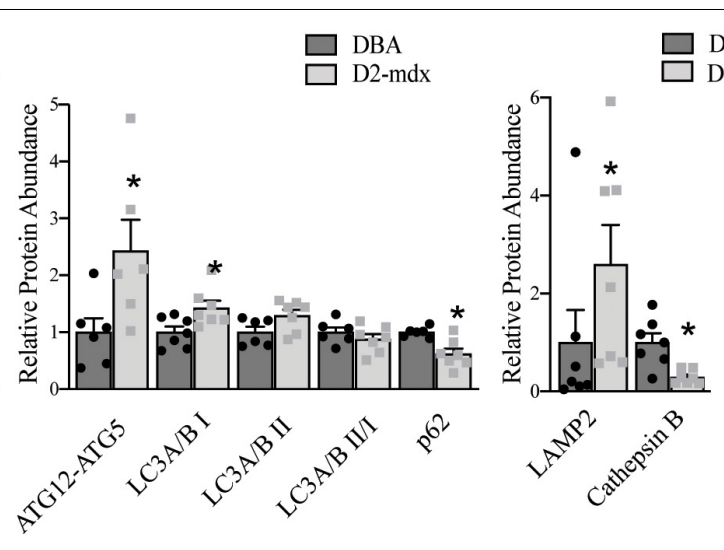

DBA D2 DBA D2

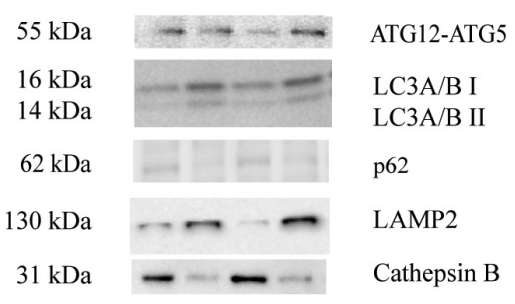

Ponceau Stain DBA D2 DBAD2

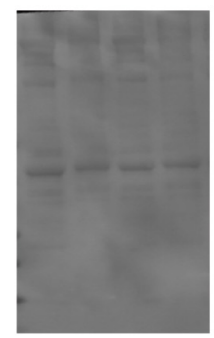

FIGURE 2 | Increased autophagosome degradation in gastrocnemius from D2-mdx. Western blot analysis of autophagy markers in whole muscle extract from 11 month old D2-mdx and DBA gastrocnemius. (A) Among the autophagy activation markers, AMPK, pAMPK T172, Beclin1 and pBeclin1 S91 were increased in dystrophic muscle compared to DBA whereas pULK1 S555 was reduced. The autophagosome formation marker ATG12-ATG5 was increased in D2-mdx gastrocnemius muscle compared to muscle from DBA. LC3I was increased and p62 was reduced in D2-mdx gastrocnemius muscle. The lysosomal marker LAMP2 was increased and lysosomal enzyme Cathepsin B was decreased in muscle from D2-mdx compared to DBA. (B) Representative western blot images and corresponding Ponceau stain image. Significance is established at $p<0.05$. * Indicates significance between D2-mdx and DBA ( $n=7$ mice per group).

in gastrocnemius from D2-mdx mice compared to DBA and pBeclin1 S91/Beclin1 was similar between groups (Figure 2). Autophagosome formation marker ATG12-ATG5 was increased in diseased muscle by 2.5 -fold ( $p<0.05$; Figure 2). LC3A/B I was increased 1.4-fold ( $p<0.05$ ), but the lipidated form, LC3A/B II, as well as the ratio LC3A/B II/I were similar between healthy and diseased muscles (Figure 2). p62 was reduced by $40 \%(p<0.01)$ in gastrocnemius from D2-mdx compared to DBA (Figure 2). LAMP2 was increased by 2.5 -fold $(p<0.05)$ in dystrophic muscles and Cathepsin $\mathrm{B}$, a lysosomal cysteine protease, was reduced by approximately $75 \%(p<0.01)$ in D2-mdx (Figure 2).

\section{Cytosolic and Lysosome Enriched Fractions}

The findings in whole muscle extract from gastrocnemius did not support our expectation of decreased autophagosome degradation and lysosomal abundance. To better understand how autophagy may be altered by dystrophin deficiency in gastrocnemius from the D2-mdx model and to better appreciate lysosome-mediated degradation of autophagosomes, we separated the gastrocnemius into a lysosome enriched fraction (LEF) and a cytosolic fraction (CF). In the CF total AMPK, pAMPK T172, and pAMPK T172/total AMPK were similar between groups (Figure 3). Relative protein abundance of ULK1 was decreased by 70\% $(p<0.0001)$ and pULK1 S555 was decreased by $80 \%(p<0.001)$ in dystrophindeficient muscle compared to healthy muscle (Figure 3). Similarly, total Beclin1 and pBeclin1 (phosphorylated at S91) were decreased by approximately 55\% $(p<0.0001)$ in dystrophic muscle compared to healthy muscle (Figure 3). Because of the similar reduction in total and phosphorylated proteins, the ratios of pULK1 S555/total ULK1 and pBeclin1 S91/total Beclin1 were similar between groups (Figure 3). Relative protein abundance of PI3K III was increased by $40 \%(p<0.05)$ in CF from D2-mdx compared to CF from DBA (Figure 3).

The autophagosome formation markers were only detected in the CF in healthy and dystrophic muscles. In the CF, we discovered that ATG16L1 was decreased by 63\% in dystrophic muscle compared to healthy ( $p<0.001$; Figure 4). ATG12, ATG12-ATG5 complex, and the ratio between the ATG12-ATG5 complex and ATG12 were similar between groups (Figure 4).

LC3A/B I and LC3A/B II were detected in both the CF and LEF (Figure 5). Relative protein abundance of LC3A/B I was similar between fractions and between groups (Figure 5). LC3A/B II was significantly decreased in CF compared to LEF but was similar as a main effect of disease (Figure 5). LC3A/B II/I was decreased 


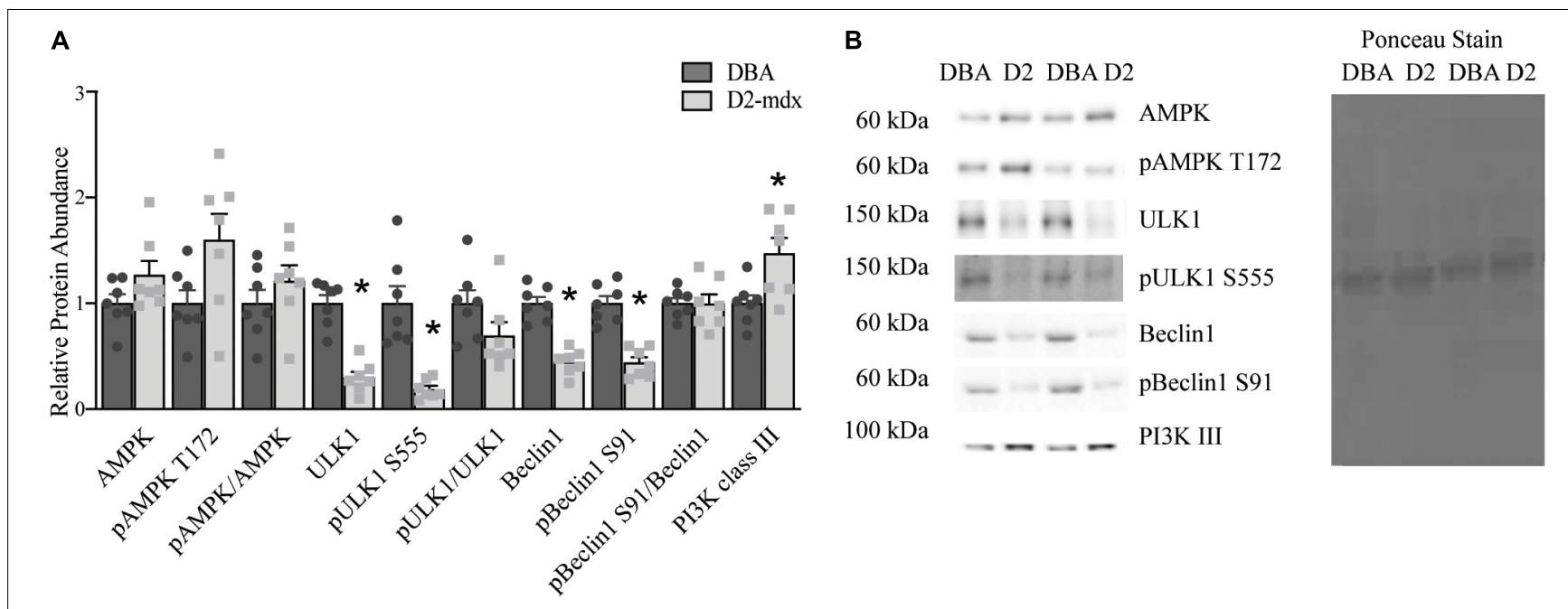

FIGURE 3 | Markers of autophagic activation analyzed in a cytosolic fraction from gastrocnemius muscles. Western blot analysis of activation markers of autophagy in a cytosolic fraction from gastrocnemius muscles from 11 month old D2-mdx and DBA mice. (A) ULK1, pULK1 S555, Beclin1and pBeclin1 S91 were decreased, while PI3K III was elevated in D2-mdx gastrocnemius cytoplasmic fraction (CF) compared to DBA. (B) Representative western blot images and corresponding Ponceau stain image. Significance is established at $p<0.05$. ${ }^{*}$ Indicates significance between D2-mdx and DBA ( $n=7$ mice per group).

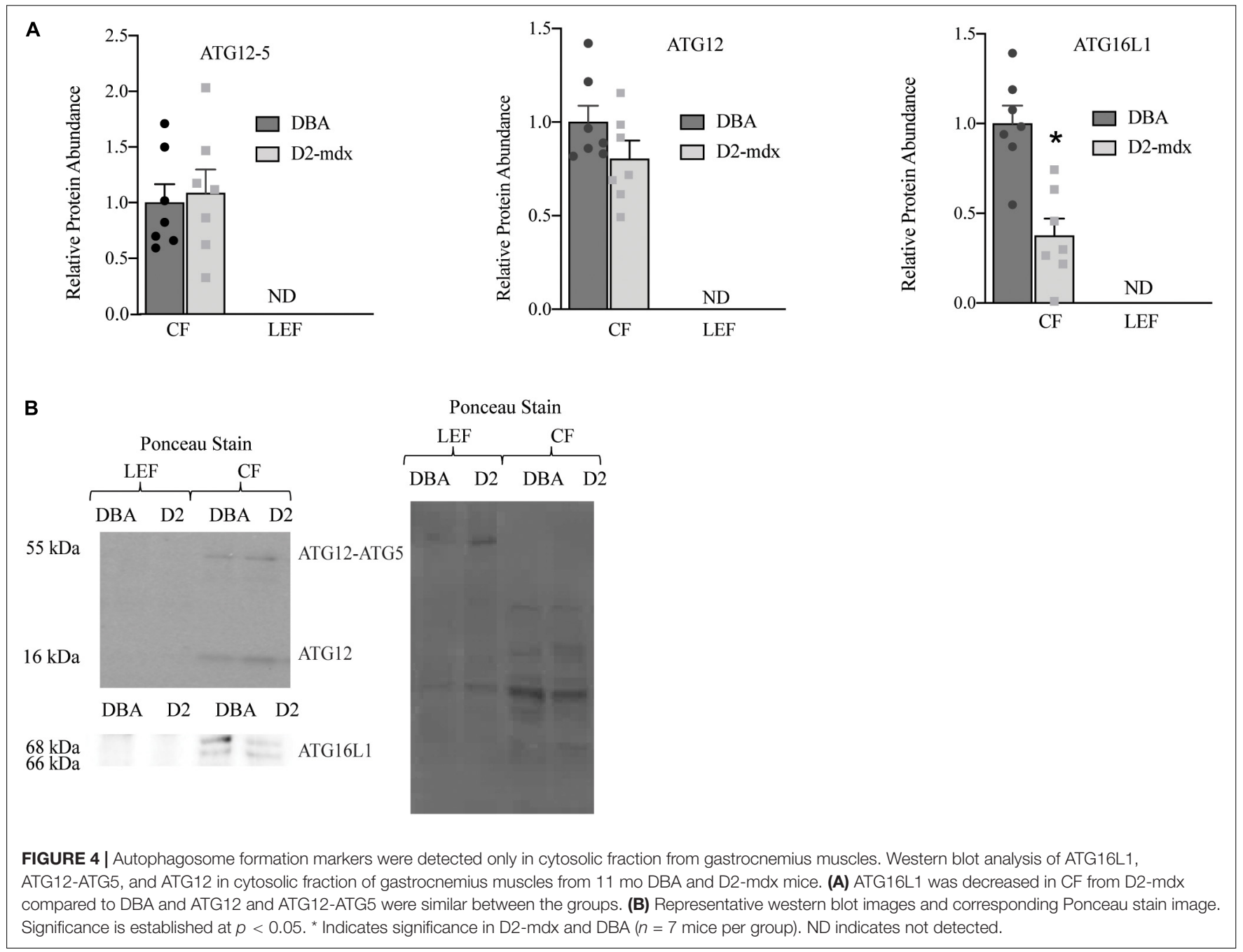




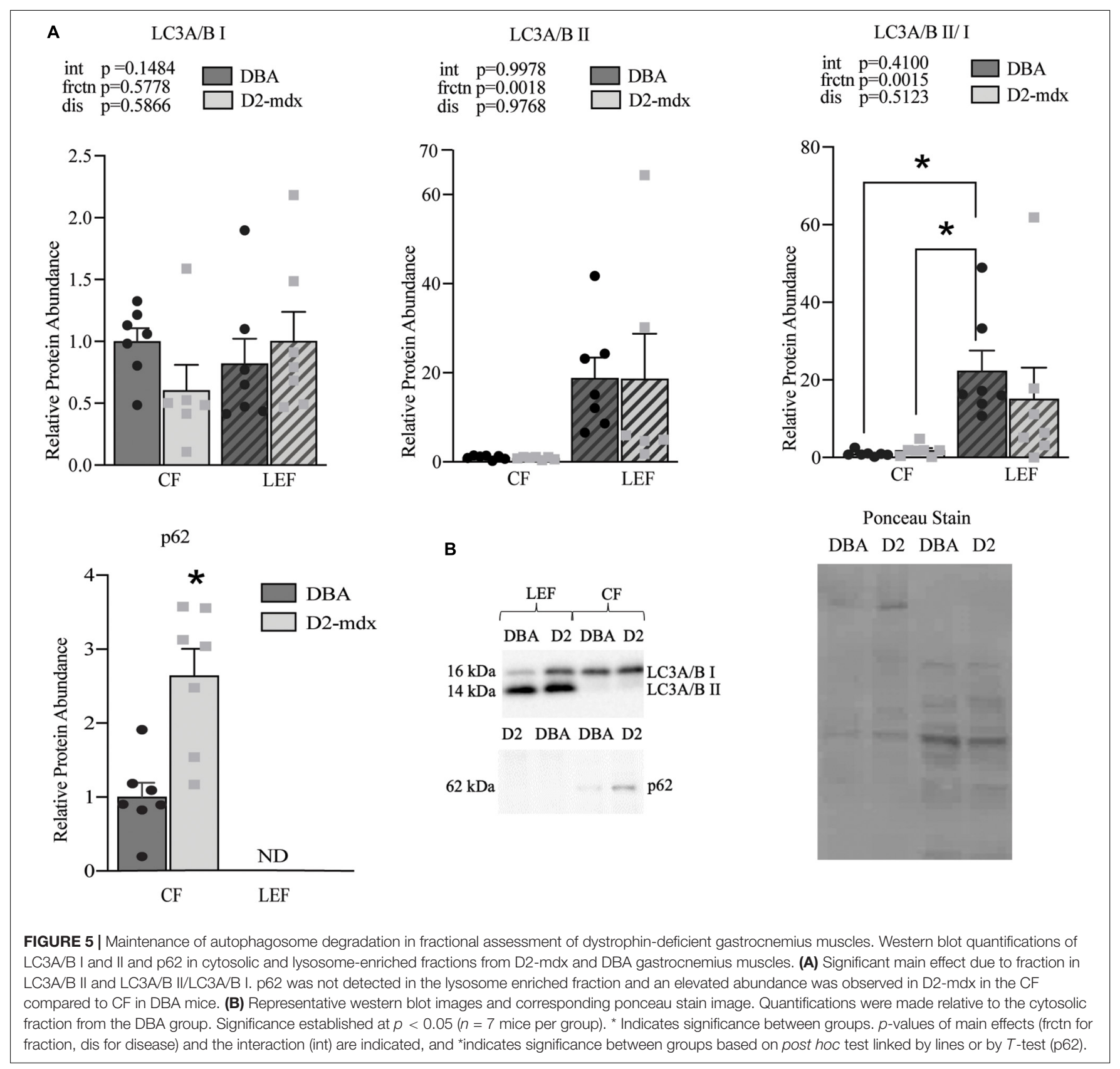

in LEF as a main effect of fraction but was similar as a main effect of disease (Figure 5). p62 was only detectable in the CF, suggesting a complete degradation of p62 within lysosomes in both healthy and diseased muscles (Figure 5). In the CF, relative protein abundance of $\mathrm{p} 62$ was increased 2.6-fold $(p<0.01)$ in D2-mdx compared to DBA (Figure 5).

The lysosomal membrane protein marker LAMP2 was used to confirm effective isolation of a LEF and was detected only in the LEF from DBA and D2-mdx (Figure 6). LAMP2 was increased by 3 -fold $(p<0.05)$ in dystrophic muscle compared to healthy muscle (Figure 6). Cathepsin B, a lysosomal enzyme, was detected in both fractions. Of note, while Cathepsin B was detected in both fractions, the banding patterns differed substantially between the CF and the LEF, further confirming fraction isolation. Interestingly, the $31 \mathrm{kDa}$ band (single chain mature Cathepsin B (Mort and Buttle, 1997; Cavallo-Medved et al., 2017) was detected only in the LEF for both DBA and D2$\mathrm{mdx}$ and was similar between groups (Figure 6). The $26 \mathrm{kDa}$ band, which represents the heavy chain of mature Cathepsin B protein (Mort and Buttle, 1997; Cavallo-Medved et al., 2017), was found both in the LEF as well as CF (Figure 6). There was a significant main effect of fraction such that the relative abundance was decreased by approximately $50 \%$ in LEF compared to the CF. Further, there was a main effect of disease such that D2mdx was decreased by $50 \%$ compared to DBA (Figure 6). In the $\mathrm{CF}$, the relative protein abundance was decreased by 


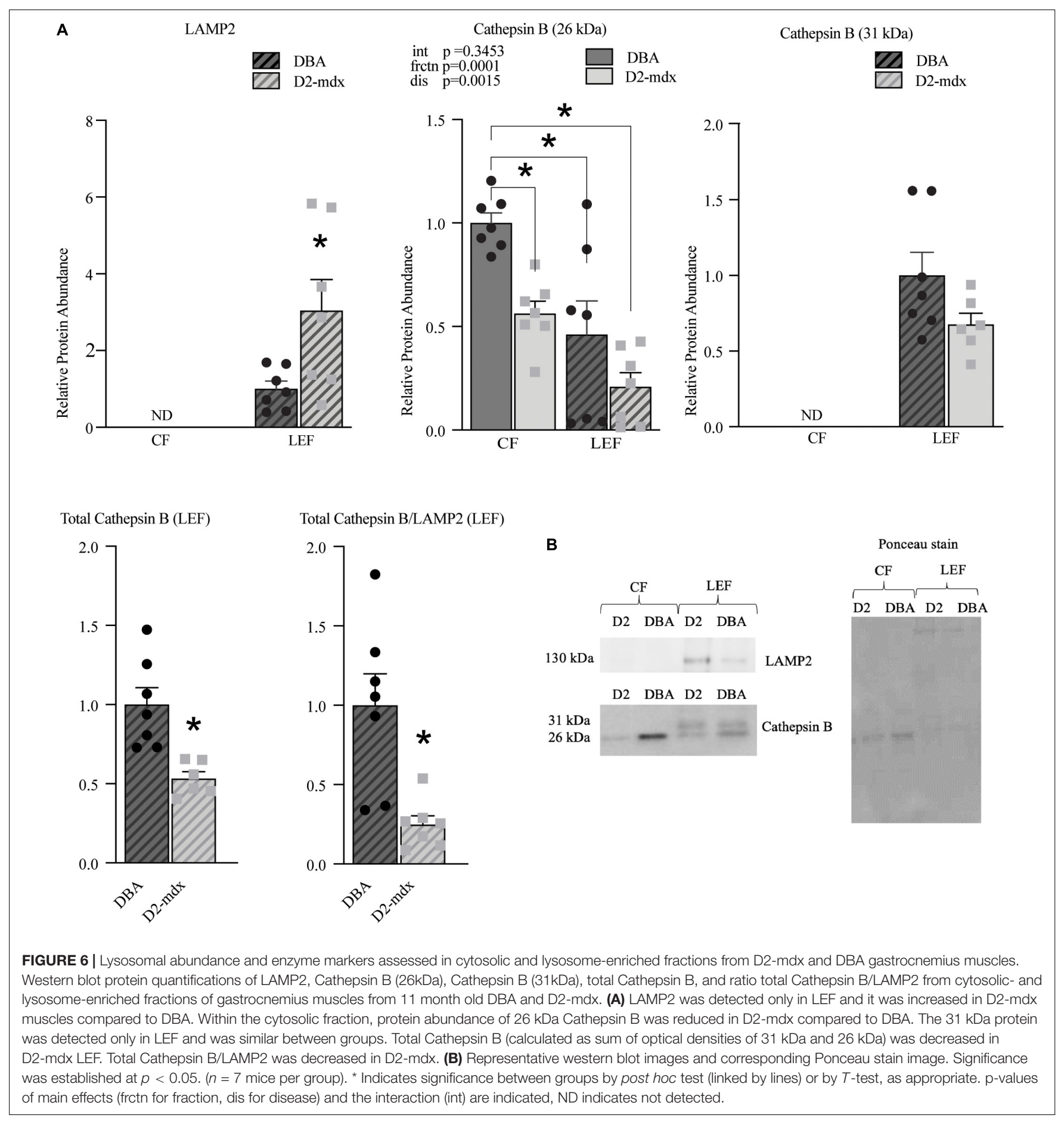

approximately $50 \%$ in dystrophic muscle compared to healthy muscle $(p<0.05$; Figure 6). Since the banding pattern differed between the fractions, a sum of the optical density of the $26 \mathrm{kDa}$ and $31 \mathrm{kDa}$ bands in the LEF was considered as total Cathepsin B in this fraction. Total Cathepsin B was decreased by approximately 50\% $(p<0.01)$ in dystrophic muscle compared to healthy muscle (Figure 6). To gain preliminary insight into lysosomal health we expressed Cathepsin B relative to LAMP2.
Here, Cathepsin B was considered as a proxy for function since it is a lysosomal cysteine protease that contributes to degradation inside lysosomes. LAMP2 was used as an indicator of lysosomal abundance as we and others have done previously (Iwata et al., 2005; Spaulding et al., 2018; Klionsky et al., 2021). Given the known role of each protein we reasoned that the ratio of Cathepsin B to LAMP2 would provide additional insight into the health of the total lysosomal pool. We discovered that this ratio 
was decreased by $75 \%(p<0.01)$ in dystrophic muscle compared to healthy muscle (Figure 6).

\section{DISCUSSION}

Duchenne muscular dystrophy is a fatal muscle degenerative disease caused by the absence of a functional dystrophin protein. Among the various cellular dysfunctions associated with DMD, upstream activation of autophagy and degradation of autophagosomes appear to be suppressed in skeletal muscles from the mdx mouse model (De Palma et al., 2012; Pauly et al., 2012; Spitali et al., 2013; Spaulding et al., 2018). Limited data from human DMD patients also indicate defective autophagy in skeletal muscle (De Palma et al., 2012; Fiacco et al., 2016). Conversely, autophagy appears to be stimulated in dystrophic hearts from rodent models, despite histopathological injury; a fact that may highlight nuanced pathology in chronically active cardiac muscle (Kang et al., 2018; Spaulding et al., 2019a). In support, cardioprotective effects were observed by treating mdx mice with rapamycin nanoparticles, and suggested amelioration of defective autophagy in dystrophic hearts (Bibee et al., 2014). Since the $\mathrm{mdx}$ model displays a mild disease phenotype, others have argued the more severe D2-mdx model may better recapitulate disease (Coley et al., 2016; van Putten et al., 2019) and, therefore, may more accurately predict therapeutic success in clinical trials (Hammers et al., 2020). However, how dystrophin deficiency alters autophagy in the D2-mdx model is not well-understood. We hypothesized that autophagosome degradation and lysosome abundance would be decreased in muscles from D2-mdx mice compared to muscle from DBA mice. This hypothesis was largely supported in the diaphragm as degradation of autophagosomes appeared to be suppressed. However, counter to our hypothesis, degradation of autophagosomes appeared to be stimulated in the gastrocnemius potentially due to an expansion of the lysosomal pool.

Autophagy is a multistep cellular process through which cells degrade and recycle damaged and dysfunctional cellular contents. Multiple proteins regulate activation, autophagosome formation and maturation, and degradation (Parzych and Klionsky, 2014). Canonical activation of autophagy was not robustly stimulated in diaphragm or gastrocnemius muscles, however, we identified muscle-dependent expression of some activation markers. In this experiment we also discovered elevation of pBeclin1, particularly in diaphragm, which may lead to a non-canonical activation of autophagy (Wang et al., 2009; Zhou et al., 2013). Despite the absence of upstream activation, markers of autophagosome formation and maturation were elevated in dystrophic diaphragm and gastrocnemius muscles compared to healthy muscle. These data are in good agreement with our previous work that showed elevated markers of autophagosome formation in mdx mice regardless of age (Spaulding et al., 2018).

While activation of autophagy and maturation of autophagosomes were similar between the diaphragm and gastrocnemius, degradation was substantially different. In the diaphragm, increased LC3A/B II along with increased p62 is consistent with impaired degradation of autophagosomes, while in the gastrocnemius similar LC3A/B II and decreased p62 is supportive of increased degradation of autophagosomes (Ichimura and Komatsu, 2010). Maintenance of autophagosome degradation in limb muscle is associated with attenuated functional decline in limb muscles (soleus and EDL) compared to diaphragms all from these animals (Spaulding et al., 2019b, 2020b). The mechanism underlying this fundamental difference is beyond the scope of this investigation, though speculatively may be influenced by the different activation patterns of these muscles, exposure to different forces, their differing fiber types, and/or differing metabolic profiles (Silverman and Atwood, 1980; Parry and Parslow, 1981; Petrof et al., 1993a; Zardini and Parry, 1994; Augusto et al., 2004), compounded by different rates of disease progression (Hammers et al., 2020). While muscle-dependent differences in autophagy have been previously reported, to our knowledge, the mechanism underlying these differences has not been fully elucidated (Mizushima et al., 2004; Raben et al., 2008; Ogata et al., 2010; Mofarrahi et al., 2013; Fernando et al., 2020).

In a previous experiment we discovered that lysosome abundance was decreased in dystrophic diaphragms with advanced disease (Spaulding et al., 2018). Independently, we also reported that stimulation of lysosomal biogenesis was associated with increased degradation of autophagosomes and decreased disease severity (Spaulding et al., 2020a). Collectively, these data indicate that impaired degradation of autophagosomes is due, at least in part, to decreased lysosomal content in dystrophic muscle from $\mathrm{mdx}$ mice. In this investigation using the D2-mdx model we found that in diaphragms with advanced disease lysosomal content was similar between groups and we identify this as a potential difference between these models, though acknowledge the differences in age of mice in our previous work and the work contained herein. Importantly though, while mice were of different ages (e.g., 17 mo old mdx mice and 11 mo old D2-mdx mice) muscles from both experiments had profound disease-related injury. Conversely, in D2-mdx gastrocnemius muscles, lysosomal abundance was elevated, which may allow maintenance of degradative power. Speculatively, decreased Cathepsin B may be suggestive of impaired lysosomal function. Hence, a larger pool of less functional lysosomes may be necessary to maintain total lysosomal function and degradation of autophagosomes; a point emphasized by findings in associated LEFs. In the gastrocnemius, increased degradation of autophagosomes contradicts findings from $\mathrm{mdx}$ mice as well as human patients, which indicate autophagosome accumulation in dystrophic muscles (De Palma et al., 2012; Pauly et al., 2012; Spitali et al., 2013; Spaulding et al., 2018, 2020a).

As maintained, or even increased, degradation of autophagosomes was unexpected in dystrophin-deficient muscle, to better understand degradation of autophagosomes in gastrocnemius muscles, the autophagy pathway was further assessed by comparing changes within CF and LEF. Consistent with findings from whole homogenate, upstream activation of autophagy and initiation of autophagosome formation did not appear to be robustly stimulated in the CF. Interestingly, 
ATG12-ATG5, ATG12, and ATG16L1 were found only in the $\mathrm{CF}$, supporting previous discoveries indicating dissociation of this complex during autophagosome maturation (Mizushima et al., 2001; Kuma et al., 2002). That p62 was found in the CF, but not the LEF, along with similar LC3A/B II in the LEF, indicates functional lysosomal degradation of autophagosomes in dystrophin-deficient (and healthy) gastrocnemius muscles. Detection of LC3A/B I in the LEF is congruent with previous findings (Yang et al., 2011). Consistent with our observations from whole homogenates, in LEF lysosome abundance was increased in dystrophic muscle compared to healthy muscle. Importantly, and supportive of isolation of an LEF, in healthy and dystrophin-deficient muscle lysosomes were only detected in the LEF. Given these outcomes it is reasonable to suggest the increased lysosomal pool, at least in part, maintained degradation of autophagosomes in dystrophic gastrocnemius muscles raising the possibility that a larger lysosomal pool was, indeed, necessary to achieve the same degree of degradation as in healthy muscles. To gain preliminary insight into lysosomal health or function we expressed total Cathepsin B abundance relative to lysosomal abundance and discovered that lysosomes from dystrophic muscle have only $25 \%$ of total Cathepsin B compared to lysosomes from healthy muscle. These data raise the possibility of impaired lysosomal function in dystrophic muscle, which is mitigated, at least in part, by a larger lysosomal pool.

There are several limitations that should be considered when interpreting data presented here in. Though cellular fractionation provides an excellent tool for lysosomal isolation, presence of some non-lysosomal components is possible (Oberle et al., 2010). This was our rationale for specific use of the term LEF, rather than lysosomal fraction. In addition, though whole muscle is composed of heterogeneous cell types, including muscle fibers, satellite cells, immune cells, endothelial cells, fibroblasts etc., the vast majority is comprised of muscle fibers (Rubenstein et al., 2020). Given the incredible disparity in muscle fiber volume, mass, and nuclei relative to other cell types it seems unlikely that interpretation of present data would be significantly impacted by changes in these other cell types. Finally, we acknowledge some differences in outcomes measured in whole homogenate and measured in CF. These differences are likely due to differences in the retained components in the whole homogenate and the $\mathrm{CF}$ and may also point to disease-related impacts on protein localization or interaction (Strappazzon et al., 2011; Seibenhener et al., 2013; Liu et al., 2016; Lan et al., 2018).

\section{REFERENCES}

Augusto, V., Padovani, C. R., and Campos, G. E. R. (2004). Skeletal muscle fiber types in C57BL6J mice. Brazil. J. Morphol. Sci. 21, 89-94.

Ballabio, A. (2016). The awesome lysosome. EMBO Mol. Med. 8, 73-76. doi: 10.15252/emmm.201505966

Bibee, K. P., Cheng, Y.-J., Ching, J. K., Marsh, J. N., Li, A. J., Keeling, R. M., et al. (2014). Rapamycin nanoparticles target defective autophagy in muscular dystrophy to enhance both strength and cardiac function. FASEB J. 28, 20472061. doi: 10.1096/fj.13-237388

Castets, P., Frank, S., Sinnreich, M., and Rüegg, M. A. (2016). Get the balance right": pathological significance of autophagy perturbation in neuromuscular disorders. J. Neuromusc. Dis. 3, 127-155. doi: 10.3233/jnd-160153

\section{CONCLUSION}

We discovered that markers of autophagy differ between diaphragms and gastrocnemius muscles from D2-mdx mice. Most substantially, in diaphragms, degradation of autophagosomes appears blunted, however, in gastrocnemius muscle degradation of autophagosomes appears to be functional due, at least in part, to an increased lysosomal pool. This notion was further supported by deeper investigation using a LEF, which also raised the possibility of a larger lysosomal pool coupled with impaired lysosomal function. In addition to providing novel insight regarding autophagic degradation in an emerging mouse model, these data indicate the need for careful consideration of disease model and muscle of interest in future studies of autophagy.

\section{DATA AVAILABILITY STATEMENT}

The original contributions presented in the study are included in the article/supplementary material, further inquiries can be directed to the corresponding author.

\section{ETHICS STATEMENT}

The animal study was reviewed and approved by the Institutional Animal Care and Use Committees at the University of Montana and the University of Florida.

\section{AUTHOR CONTRIBUTIONS}

JS and JQ designed the animal experiment. JS, HS, SK, and MH conceived the idea for this experiment and interpreted the data. Tissue and data collection, and data analysis were performed by SK, HS, JQ, and TQ. JS and SK drafted the manuscript. All authors contributed in manuscript revision and editing.

\section{FUNDING}

This work was funded in part by the Michael's Cause and Ryan's Quest (JS and JQ) as well as Parent Project Muscular Dystrophy (JS and JQ).

Cavallo-Medved, D., Moin, K., and Sloane, B. (2017). Cathepsin B. AFCS Nat .Mol. 40, 1-17. doi: 10.1016/j.physbeh.2017.03

Chen, Y. W., Nagaraju, K., Bakay, M., McIntyre, O., Rawat, R., Shi, R., et al. (2005). Early onset of inflammation and later involvement of TGF $\beta$ in Duchenne muscular dystrophy. Neurology 65, 826-834. doi: 10.1212/01.wnl.0000173836. 09176.c4

Coley, W. D., Bogdanik, L., Vila, M. C., Yu, Q., Van Der Meulen, J. H., Rayavarapu, S., et al. (2016). Effect of genetic background on the dystrophic phenotype in $\mathrm{mdx}$ mice. Hum. Mol. Genet. 25, $130-145$.

De Palma, C., Morisi, F., Cheli, S., Pambianco, S., Cappello, V., Vezzoli, M., et al. (2012). Autophagy as a new therapeutic target in Duchenne muscular dystrophy. Cell Death Dis. 3, e418-10. 
Ehmsen, J., Poon, E., and Davies, K. (2002). The dystrophin-associated protein complex. J. Cell Sci. 115, 2801-2803. doi: 10.1242/jcs.115.14.2801

Farre, J. C., and Subramani, S. (2016). Mechanistic insights into selective autophagy pathways: lessons from yeast. Nat. Rev. Mol. Cell Biol. 17, 537-552. doi: 10.1038/ nrm.2016.74

Fernando, R., Castro, P., Flore, T., Deubel, S., Grune, T., Ott, C., et al. (2020). Age-related maintenance of the autophagy-lysosomal system is dependent on skeletal muscle type. Oxid. Med. Cell Longev. 2020:4908162.

Fiacco, E., Castagnetti, F., Bianconi, V., Madaro, L., De Bardi, M., Nazio, F., et al. (2016). Autophagy regulates satellite cell ability to regenerate normal and dystrophic muscles. Cell Death Differ. 23, 1839-1849. doi: 10.1038/cdd.2016.70

Hammers, D. W., Hart, C. C., Matheny, M. K., Wright, L. A., Armellini, M., Barton, E. R., et al. (2020). The D2.mdx mouse as a preclinical model of the skeletal muscle pathology associated with Duchenne muscular dystrophy. Sci. Rep. 10:14070.

Hoffman, E. P., Brown, R. H., and Kunkel, L. M. (1987). Dystrophin: the protein product of the duchenne muscular dystrophy locus. Cell 51, 919-928. doi: 10.1016/0092-8674(87)90579-4

Hollinger, K., and Selsby, J. T. (2013). The physiological response of protease inhibition in dystrophic muscle. Acta Physiol. (Oxf.) 208, 234-244. doi: 10.1111/ apha. 12114

Houang, E. M., Sham, Y. Y., Bates, F. S., and Metzger, J. M. (2018). Muscle membrane integrity in Duchenne muscular dystrophy: Recent advances in copolymer-based muscle membrane stabilizers. Skeletal Muscle 8, 1-19.

Ichimura, Y., and Komatsu, M. (2010). Selective degradation of p62 by autophagy. Semin. Immunopathol. 32, 431-436. doi: 10.1007/s00281-010-0220-1

Iwata, A., Riley, B. E., Johnston, J. A., and Kopito, R. R. (2005). HDAC6 and microtubules are required for autophagic degradation of aggregated huntingtin*. J. Biol. Chem. 280, 40282-40292. doi: 10.1074/jbc.m508786200

Kang, C., Badr, M. A., Kyrychenko, V., Eskelinen, E. L., and Shirokova, N. (2018), Deficit in PINK1/PARKIN-mediated mitochondrial autophagy at late stages of dystrophic cardiomyopathy. Cardiovasc. Res. 114, 90-102. doi: 10.1093/cvr/ cvx201

Klionsky, D. J., Abdel-Aziz, A. K., Abdelfatah, S., Abdellatif, M., Abdoli, A., Abel, S., et al. (2021). Guidelines for the use and interpretation of assays for monitoring autophagy (4th edition). Autophagy 17, 1-382.

Kocaturk, N. M., and Gozuacik, D. (2018). Crosstalk between mammalian autophagy and the ubiquitin-proteasome system. Front. Cell Dev. Biol. 6:128. doi: 10.3389/fcell.2018.00128

Kuma, A., Mizushima, N., Ishihara, N., and Ohsumi, Y. (2002). Formation of the $\mathrm{j} 350-\mathrm{kDa}$ Apg12-Apg5 $\frac{1}{7}$ Apg16 multimeric complex, mediated by apg16 oligomerization, is essential for autophagy in yeast *. J. Biol. Chem. 277, 18619-18625. doi: 10.1074/jbc.m111889200

Lan, R., Wu, J.-T., Wu, T., Ma, Y.-Z., Wang, B.-Q., Zheng, H.-Z., et al. (2018). Mitophagy is activated in brain damage induced by cerebral ischemia and reperfusion via the PINK1/Parkin/p62 signalling pathway. Brain Res. Bull. 142, 63-77. doi: 10.1016/j.brainresbull.2018.06.018

Liu, W. J., Ye, L., Huang, W. F., Guo, L. J., Xu, Z. G., Wu, H. L., et al. (2016). p62 links the autophagy pathway and the ubiqutin-proteasome system upon ubiquitinated protein degradation. Cell Mol. Biol. Lett. 21:29.

Ljubicic, V., Khogali, S., Renaud, J. M., and Jasmin, B. J. (2012). Chronic AMPK stimulation attenuates adaptive signaling in dystrophic skeletal muscle. Am. J. Physiol. Cell Physiol. 302, 110-121.

Mizushima, N., Yamamoto, A., Hatan, M., Kobayashi, Y., Kabeya, Y., Suzuki, K., et al. (2001). Dissection of autophagosome formation using Apg5-deficient mouse embryonic stem cells. J. Cell Biol. 152, 657-667. doi: 10.1083/jcb.152. 4.657

Mizushima, N., Yamamoto, A., Matsui, M., Yoshimori, T., and Ohsumi, Y. (2004). In vivo analysis of autophagy in response to nutrient starvation using transgenic mice expressing a fluorescent autophagosome marker. Mol. Biol. Cell 15, 11011111. doi: 10.1091/mbc.e03-09-0704

Mofarrahi, M., Guo, Y., Haspel, J. A., Choi, A. M., Davis, E. C., Gouspillou, G., et al. (2013). Autophagic flux and oxidative capacity of skeletal muscles during acute starvation. Autophagy 9, 1604-1620. doi: 10.4161/auto.25955

Morris, C. A., Selsby, J. T., Morris, L. D., Pendrak, K., and Sweeney, H. L. (2010). Bowman-birk inhibitor attenuates dystrophic pathology in mdx mice. J. Appl. Physiol. (Bethesda MD 1985) 109, 1492-1499. doi: 10.1152/japplphysiol.01283. 2009
Mort, J. S., and Buttle, D. J. (1997). Cathepsin B. Int. J. Biochem. Cell Biol. 29, 715-720.

Oberle, C., Huai, J., Reinheckel, T., Tacke, M., Rassner, M., Ekert, P. G., et al. (2010). Lysosomal membrane permeabilization and cathepsin release is a Bax/Bakdependent, amplifying event of apoptosis in fibroblasts and monocytes. Cell Death Differ. 17, 1167-1178. doi: 10.1038/cdd.2009.214

Ogata, T., Oishi, Y., and Higuchi, M. I (2010). Muraoka, fasting-related autophagic response in slow- and fast-twitch skeletal muscle. Biochem. Biophys. Res. Commun. 394, 136-140. doi: 10.1016/j.bbrc.2010.02.130

Pal, R., Palmieri, M., Loehr, J. A., Li, S., Abo-Zahrah, R., Monroe, T. O., et al. (2015). Src-dependent impairment of autophagy by oxidative stress in a mouse model of Duchenne muscular dystrophy. Nat. Commun. 5, 1-10.

Parry, D. J., and Parslow, H. G. (1981). Fiber type susceptibility in dystrophic mouse. Exp. Neurol. 73, 674-685. doi: 10.1016/0014-4886(81)90204-1

Parzych, K. R., and Klionsky, D. J. (2014). An overview of autophagy: morphology, mechanism, and regulation. Antioxid. Redox Signal. 20, 460-473. doi: 10.1089/ ars.2013.5371

Pauly, M., Daussin, F., Burelle, Y., Li, T., Godin, R., Fauconnier, J., et al. (2012). AMPK activation stimulates autophagy and ameliorates muscular dystrophy in the mdx mouse diaphragm. Am. J. Pathol. 181, 583-592. doi: 10.1016/j.ajpath. 2012.04.004

Petrof, B. J., Shrager, J. B., Stedman, H. H., Kelly, A. M., and Sweeney, H. L. (1993b). Dystrophin protects the sarcolemma from stresses developed during muscle contraction. Proc. Natl. Acad. Sci. U.S.A. 90, 3710-3714. doi: 10.1073/pnas.90. 8.3710

Petrof, B. J., Stedman, H. H., Shrager, J. B., Eby, J., Sweeney, H. L., Kelly, A. M., et al. (1993a). Adaptations in myosin heavy chain expression and contractile function in dystrophic mouse diaphragm. Am. J. Physiol. 265, C834-C841.

Raben, N., Hill, V., Shea, L., Takikita, S., Baum, R., Mizushima, N., et al. (2008). Suppression of autophagy in skeletal muscle uncovers the accumulation of ubiquitinated proteins and their potential role in muscle damage in Pompe disease. Hum. Mol. Genet. 17, 3897-3908. doi: 10.1093/hmg/ddn292

Rahimov, F., and Kunkel, L. M. (2013). Cellular and molecular mechanisms underlying muscular dystrophy. J. Cell Biol. 201, 499-510. doi: 10.1083/jcb. 201212142

Roczniak-Ferguson, A., Petit, C. S., Froehlich, F., Qian, S., Ky, J., Angarola, B., et al. (2012). The transcription factor TFEB links mTORC1 signaling to transcriptional control of lysosome homeostasis. Sci. Signal. 5:ra42. doi: 10. 1126/scisignal.2002790

Rubenstein, B., Smith, G. R., Raue, U., Begue, G., Minchev, K., Ruf-Zamojski, F., et al. (2020). Single-cell transcriptional profiles in human skeletal muscle. Sci. Rep. 10:229.

Saftig, P., and Klumperman, J. (2009). Lysosome biogenesis and lysosomal membrane proteins: trafficking meets function. Nat. Rev. Mol. Cell Biol. 10, 623-635. doi: $10.1038 / \mathrm{nrm} 2745$

Sandri, M. (2010). Autophagy in skeletal muscle. FEBS Lett. 584, 1411-1416. doi 10.1016/j.febslet.2010.01.056

Seibenhener, M. L., Du, Y., Diaz-Meco, M. T., Moscat, J., Wooten, M. C., Wooten, M. W., et al. (2013). A role for sequestosome $1 / \mathrm{p} 62$ in mitochondrial dynamics, import and genome integrity. Biochim. Biophys. Acta (BBA) Mol. Cell Res. 1833 , 452-459. doi: 10.1016/j.bbamcr.2012.11.004

Selsby, J., Morris, C., Morris, L., and Sweeney, L. (2012). A proteasome inhibitor fails to attenuate dystrophic pathology in $\mathrm{mdx}$ mice. PLoS Curr. 4:e4f84a944d8930. doi: 10.1371/4F84A944D8930

Selsby, J., Pendrak, K., Zadel, M., Tian, Z., Pham, J., Carver, T., et al. (2010). Leupeptin-based inhibitors do not improve the mdx phenotype. Am. J. Physiol. Regul. Integr. Comp. Physiol. 299, R1192-R1201.

Selsby, J. T. (2011). Increased catalase expression improves muscle function in $\mathrm{mdx}$ mice. Exp. Physiol. 96, 194-202. doi: 10.1113/expphysiol.2010.054379

Settembre, A., and Ballabio, A. (2011). TFEB regulates autophagy: an integrated coordination of cellular degradation and recycling processes. Autophagy 7 , 1379-1381. doi: 10.4161/auto.7.11.17166

Silverman, H., and Atwood, H. L. (1980). Increase in oxidative capacity of muscle fibers in dystrophic mice and correlation with overactivity in these fibers. Exp. Neurol. 68, 97-113. doi: 10.1016/0014-4886(80)90069-2

Spaulding, H. R., Ballmann, C., Quindry, J. C., Hudson, M. B., and Selsby, J. T. (2019a). Autophagy in the heart is enhanced and independent of disease 
progression in mus musculus dystrophinopathy models. JRSM Cardiovasc. Dis. 8:2048004019879581.

Spaulding, H. R., Kelly, E. M., Quindry, J. C., Sheffield, J. B., Hudson, M. B., and Selsby, J. T. (2018). Autophagic dysfunction and autophagosome escape in the mdx mus musculus model of Duchenne muscular dystrophy. Acta Physiol. 222, $1-11$.

Spaulding, H. R., Ludwig, A. K., Hollinger, K., Hudson, M. B., and Selsby, J. T. (2020a). PGC-1 $\alpha$ overexpression increases transcription factor EB nuclear localization and lysosome abundance in dystrophin-deficient skeletal muscle. Physiol. Rep. 8, 1-9.

Spaulding, H. R., Quindry, T., Hammer, K., Quindry, J. C., and Selsby, J. T. (2019b). Nutraceutical and pharmaceutical cocktails did not improve muscle function or reduce histological damage in D2-mdx mice. J. Appl. Physiol. 127, 1058-1066. doi: 10.1152/japplphysiol.00162.2019

Spaulding, H. R., Quindry, T., Quindry, J. C., and Selsby, J. T. (2020b). Nutraceutical and pharmaceutical cocktails did not preserve diaphragm muscle function or reduce muscle damage in D2-mdx mice. Exp. Physiol. 105, 989-999. doi: $10.1113 /$ ep087887

Spitali, P., Grumati, P., Hiller, M., Chrisam, M., Aartsma-Rus, A., Bonaldo, P., et al. (2013). Autophagy is impaired in the tibialis anterior of dystrophin null mice. PLoS Curr. 5:ecurrents.md.e1226cefa851a2f079bbc406c0a21e80. doi: 10.1371/ currents.md.e1226cefa851a2f079bbc406c0a21e80

Strappazzon, F., Vietri-Rudan, M., Campello, S., Nazio, F., Florenzano, F., Fimia, G. M., et al. (2011). Mitochondrial BCL-2 inhibits AMBRA1-induced autophagy. EMBO J. 30, 1195-1208. doi: 10.1038/emboj.2011.49

van Putten, M., Putker, K., Overzier, M., Adamzek, W. A., Pasteuning-Vuhman, S., Plomp, J. J., et al. (2019). Natural disease history of the D2-mdx mouse model for Duchenne muscular dystrophy. FASEB J. 33, 8110-8124. doi: 10.1096/fj. $201802488 \mathrm{r}$
Wang, J., Whiteman, M. W., Lian, H., Wang, G., Singh, A., Huang, D., et al. (2009). A non-canonical MEK/ERK signaling pathway regulates autophagy via regulating Beclin 1. J. Biol. Chem. 284, 21412-21424. doi: 10.1074/jbc.m109. 026013

Yang, D. S., Stavrides, P., Mohan, P. S., Kaushik, S., Kumar, A., Ohno, M., et al. (2011). Reversal of autophagy dysfunction in the TgCRND8 mouse model of Alzheimer's disease ameliorates amyloid pathologies and memory deficits. Brain 134, 258-277. doi: 10.1093/brain/awq341

Zardini, D. M., and Parry, D. J. (1994). Identification, distribution, and myosin subunit composition of type IIX fibers in mouse muscles. Muscle Nerve 17, 1308-1316. doi: 10.1002/mus.880171110

Zhou, L., Wang, H. F., Ren, H. G., Chen, D., Gao, F., Hu, Q. S., et al. (2013). Bcl2 -dependent upregulation of autophagy by sequestosome 1/p62 in vitro. Acta Pharmacol. Sin. 34, 651-656. doi: 10.1038/aps.2013.12

Conflict of Interest: JS and MH are founders of Extrave Bioscience, LLC. This work does not have any obvious connection to goals of Extrave Bioscience, LLC.

The remaining authors declare that the research was conducted in the absence of any commercial or financial relationships that could be construed as a potential conflict of interest.

Copyright (c) 2021 Krishna, Spaulding, Quindry, Hudson, Quindry and Selsby. This is an open-access article distributed under the terms of the Creative Commons Attribution License (CC BY). The use, distribution or reproduction in other forums is permitted, provided the original author(s) and the copyright owner(s) are credited and that the original publication in this journal is cited, in accordance with accepted academic practice. No use, distribution or reproduction is permitted which does not comply with these terms. 